\title{
Book Review-Diagnostic pathology: molecular oncology, 1st edition
}

\author{
Daniela Massi $^{1}$
}

Received: 4 June 2016 / Accepted: 5 June 2016 / Published online: 24 June 2016

(C) Springer-Verlag Berlin Heidelberg 2016

Personalized medicine, or precision medicine, is becoming a major challenge for scientists and physicians, and a great matter of hope for patients. The genetic and molecular bases of such an approach are rapidly changing and offering a new way of establishing diagnoses and designing treatments. This novel era has already started with a robust impact, particularly in clinical oncology. Personalized therapy for patients whose tumors have been characterized by pathologists by molecular tools is currently practiced for hematological tumors, breast, lung, skin, and many other cancers. Thus, molecular oncologic pathology may be defined as one of the most dynamic areas of medicine.

The nine sections of the 936-page book "Diagnostic Pathology: Molecular Oncology, 1st Edition" associate clinical and morphological features of hematopoietic and lymphoid neoplasms and solid tumors with the genetic and molecular biomarkers that orient pathologist in the most updated diagnosis, and hematologists and oncologists to the most precise and targeted treatment.

The schematic and concise format facilitates greatly the consultation, and I am convinced that some hints will be particularly appreciated by the readers. These include the introductory description of the current techniques for DNA and chromosome analysis, a clear iconography (with more than 2000 images), and a final part dedicated to quality assurance and regulatory issues. It is easy to appreciate how important these topics can be in the current clinical practice of a reliable molecular pathology laboratory.

Finally, the contribution of a large series of outstanding colleagues-leading authorities in their own field of study and practice-warrants the required deepening in each of the topics. For each topic, emphasis is given to the integration of helpful diagnostic clues for accurate interpretation (in bulleted format) as well as relevant and appropriate molecular testing to guide clinical decision. Readers will have the chance to understand how to interpret newly found mutations and will be guided in the choice of analytical methods.

The book should be found on the desk of any pathologist, but particularly the young pathologist who aims to continue their career with the necessary curiosity for the novel world of molecular diagnoses that provide up-to-date information and the right answers that their patients need.

Mohammad A. Vasef and Aaron Auerbach (Eds.)

Print ISBN 9780323376785

$968 \mathrm{pp}$

$£ 211.99$

http://www.elsevierhealth.co.uk/diagnostic-pathologymolecular-oncology-9780323376785.html

Daniela Massi

Daniela.massi@unifi.it

1 Division of Pathology, Department of Surgery and Translational Medicine, University of Florence, Florence, Italy 